\title{
AUTONOMOUS UNDERWATER VEHICLES: SINGULAR EXTREMALS AND CHATTERING
}

\author{
M. Chyba ${ }^{1}$ and T. Haberkorn ${ }^{1}$ \\ ${ }^{1}$ Department of Mathematics, University of Hawaii, Honolulu, Hawaii 96822 , \\ $\{$ mchyba,haberkor\}@math.hawaii.edu*
}

\begin{abstract}
In this paper, we consider the time minimal problem for an Autonomous Underwater Vehicle. We investigate, on a simplified model, the existence of singular extremals and discuss their optimality status. Moreover, we prove that singular extremals corresponding to the angular acceleration are of order 2 . We produce in this case a semi-canonical form of our Hamiltonian system and we can conclude the existence of chattering extremals..
\end{abstract}

keywords: Underwater Vehicles, Time optimal, Singular Extremals, Chattering.

\section{Introduction}

In this paper, we consider the time minimal problem for autonomous underwater vehicle. We first describe the general model and give the equations of motion. However, due to the space limitation of this paper, and to highlight the properties of our system without tedious and lengthly calculations, we restrict ourselves to a simplified model. The general situation will be described in a forthcoming article. Based on the maximum principle we define bang-bang and singular trajectories. A first analysis of the singular extremals of the simplified model has been done in [2] and the existence of chattering extremals for a given pair of initial and final configurations at rest was discussed in [4] using numerical computations. In this paper, first we extend the previous results under the assumption that the vehicle is symmetric, in particular we show that if an extremal is bang-bang with respect to one of the linear velocities but singular in the two other controls then it cannot be time optimal. Moreover, the major addition to our previous results is that we provide a semi-canonical form in the situation of a singular extremal with respect to the angular velocity and bang with respect to the linear velocities. From [13] the existence of chat-

*Authors supported by NSF grant DMS-030641

Please use the following format when citing this chapter:

Chyba, M., and Haberkorn, T., 2006, in IFIP International Federation for Information Processing, Volume 202, Systems, Control, Modeling and Optimization, eds. Ceragioli, F., Dontchev, A., Furuta, H., Marti, K., Pandolfi, L., (Boston:

Springer), pp. 103-113. 
tering extremals in our problem follows. We supplement our conclusions with numerical computations.

\section{The Model}

We consider a 6 degrees of freedom Autonomous Underwater Vehicle (AUV). We denote by $\eta=(x, y, z, \phi, \theta, \psi)^{t}$ the position and orientation of the vehicle in the earth-fixed reference frame, where $(\phi, \theta, \psi)$ are the classical Euler angles. The velocities $\nu=(u, v, w, p, q, r)^{t}$ are taken with respect to the body-fixed frame (see Figure 1). In the sequel $\chi$ represents the state and velocity variables: $\chi=(\eta, \nu)$.

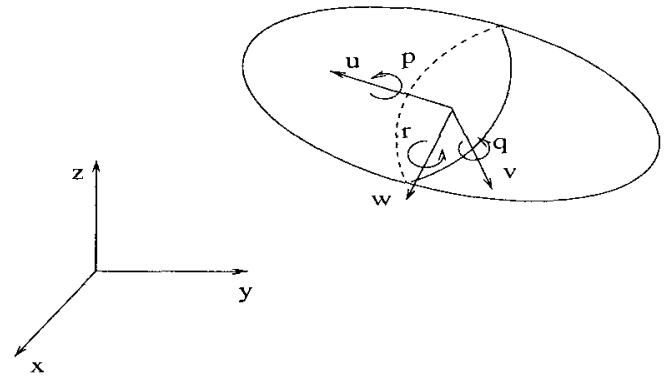

Figure 1. Earth-fixed and body-fixed frame

With those notations, the equations of motion can be written as, see [7]:

$$
\begin{aligned}
& \dot{\eta}=J(\eta) \nu \\
& \dot{\nu}=M^{-1}(\tau-[C(\nu)+D(\nu)] \nu-g(\eta))
\end{aligned}
$$

where $J(\eta)$ is the transformation matrix between the earth-fixed coordinate system and the body-fixed one. $M$ is the inertia matrix of the vehicle. The matrix $C$ takes into account for the Coriolis and centrifugal forces and is assumed to be skew-symmetric. The matrix D stands for the damping forces. The column vector $g$ represents the restoring forces and moment: gravity and buoyancy. Finally, $\tau$ is the control.

To reflect the fact that the thrusters have limited power, we assume:

$$
\tau \in \mathcal{U}=\left\{\tau \in \mathbf{R}^{6} \mid \alpha_{i} \leq \tau_{i} \leq \beta_{i}, i=1, \cdots, 6\right\},
$$

where $\alpha_{i}<0<\beta_{i}$. An admissible control is a measurable bounded function $\tau$ defined on some time interval $[0, T]$ such that $\tau(t) \in \mathcal{U}$ for a.e. $t \in[0, T]$. Due to the space limitation of this paper, we will restrict ourselves to a simplified model. The general situation will be studied in a forthcoming article. First, we assume the vehicle to be totally symmetric (hence $M$ is diagonal), with coinciding centers of gravity and buoyancy (hence $g$ is zero). The center of the 
body-fixed frame is taken at the center of gravity and the damping forces are neglected. Finally, we restrict the vehicle to move in the $x z$-plane. Under these assumptions, the equations of motion become:

$$
\begin{aligned}
\dot{x} & =u \cos \theta+w \sin \theta \\
\dot{z} & =w \cos \theta-u \sin \theta \\
\dot{\theta} & =q \\
\dot{u} & =-q w+\tau_{1} / m \\
\dot{w} & =u q+\tau_{3} / m \\
\dot{q} & =\tau_{5} / I
\end{aligned}
$$

where $m$ is the mass of the vehicle and $I$ is the first inertia momentum around the $y$-axis. For our numerical computations, we will assume $m=20$ and $I=0.1$. We have the following bounds on the controls:

$$
\left|\tau_{i}\right| \leq 1, i=1,3,5
$$

REMARK 1 The equations of motion for the velocity variables (6) -(8) are equivalent to the equations of motion for the control problem of rotation of a rigid body around its gravicenter assuming that the body is axially symmetric. The existence of chattering trajectories for the time optimal problem in this case has been studied in [13]. We provide here a generalization of these results by allowing translational motions to the body.

\section{The Maximum Principle}

Let $\chi_{0}, \chi_{T} \in \mathbf{R}^{6}$ be prescribed initial and final configurations for our simplified model. The maximum principle for the minimum time problem, [9], states that if $\bar{\tau}:[0, T] \rightarrow \mathcal{U}$ is an admissible time optimal control such that the corresponding trajectory $\bar{\chi}($.$) steers the vehicle from \chi_{0}$ to $\chi_{T}$, then there exists an absolutely continuous vector function $\lambda:[0, T] \rightarrow \mathbf{R}^{6}, \lambda(t) \neq 0$ for all $t$, such that, almost everywhere in $[0, T]$ we have:

$$
\begin{aligned}
& \dot{\bar{\chi}}_{i}(t)=\frac{\partial H}{\partial \lambda_{i}}(\bar{\chi}(t), \lambda(t), \bar{\tau}(t)) \\
& \dot{\lambda}_{i}(t)=-\frac{\partial H}{\partial \chi_{i}}(\bar{\chi}(t), \lambda(t), \bar{\tau}(t))
\end{aligned}
$$

for $i=1, \cdots, 6$, where

$$
H=\lambda^{t}\left[\begin{array}{c}
J(\eta) \nu \\
M^{-1}(\tau-C(\nu) \nu)
\end{array}\right]
$$


is the Hamiltonian function. Additionally, the maximum condition holds:

$$
H(\bar{\chi}(t), \lambda(t), \bar{\tau}(t))=\max _{\omega \in \mathcal{U}} H(\bar{\chi}(t), \lambda(t), \omega)
$$

Moreover, the maximum of the Hamiltonian is constant along the solutions of (9-10) and must satisfy $H(\lambda(t), \bar{\chi}(t), \bar{\tau}(t))=\lambda_{0}, \lambda_{0} \geq 0$. A solution $(\chi, \lambda, \tau)$ of the maximum principle, in the sense just stated, is called an extremal and the vector function $\lambda(\cdot)$ an adjoint vector. For our model, the Hamiltonian system (9-10) gives the following dynamics for the adjoint vector:

$$
\begin{aligned}
& \dot{\lambda}_{x}=0 \\
& \dot{\lambda}_{z}=0 \\
& \dot{\lambda}_{\theta}=-\lambda_{x}(w \cos \theta-u \sin \theta)+\lambda_{z}(u \cos \theta+w \sin \theta) \\
& \dot{\lambda}_{u}=-\lambda_{x} \cos \theta+\lambda_{z} \sin \theta-\lambda_{w} q \\
& \dot{\lambda}_{w}=-\lambda_{x} \sin \theta-\lambda_{z} \cos \theta+\lambda_{u} q \\
& \dot{\lambda}_{q}=-\lambda_{\theta}+\lambda_{u} w-\lambda_{w} u
\end{aligned}
$$

The maximization condition (12) implies that the control $\bar{\tau}$ satisfies $(i=1,3,5)$ :

$$
\tau_{i}(t)=-1 \text {, if } \varphi_{i}(t)<0 \text { and } \tau_{i}(t)=1 \text { if } \varphi_{i}(t)>0
$$

with $\varphi_{1}=\lambda_{u} / m, \varphi_{3}=\lambda_{w} / m, \varphi_{5}=\lambda_{q} / I$. Those functions are called the switching functions. If along the extremal, we have $\varphi_{i}(t) \neq 0$ almost everywhere on $[0, T]$, then the corresponding control $\tau_{i}$ takes its values in $\{-1,1\}$ and the extremal is said to be bang-bang with respect to $\tau_{i}$. However, if there exists a non trivial interval $\left[t_{1}, t_{2}\right]$ on which $\varphi_{i}(t)$ is identically zero, the control $\tau_{i}$ is said to be singular on $\left[t_{1}, t_{2}\right]$ and the corresponding extremal is called $\tau_{i}$-singular. Let $q$ be such that $\frac{d^{2 q}}{d t^{2 q}} \phi_{i}$ is the lowest order derivative in which $\tau_{i}$ appears explicitely with a nonzero coefficient. We defined $q$ as the order of the singular control $\tau_{i}$. The above definition lies on the fact that it is a well known result, see [10] for instance, that a singular control $\tau_{i}$ first appears explicitely in an even order derivative of $\phi_{i}$. Assume that the component $u_{i}$ of the control is bang-bang; then $t_{s} \in[0, T]$ is called a switching time for $u_{i}$ if, for each interval of the form $] t_{s}-\varepsilon, t_{s}+\varepsilon\left[\cap\left[t_{1}, t_{2}\right], \varepsilon>0\right.$, there is no constant $c$ such that $u_{i}(t)=c$ for almost all $t \in\left[t_{1}, t_{2}\right]$.

Optimal trajectories are usually concatenations of bang-bang pieces with singular pieces. The Maximum principle does not give any direct information about these concatenations and it is known that in some cases such a concatenation may involve chattering, see [8] for the first example of such optimal trajectories. 


\section{Singular trajectories}

In this section we focus on the singular trajectories for our model. It is a well known result, see for instance $[1,11]$, that for a fully actuated controlled mechanical systems all controls cannot be singular at the same time. In other words, we have:

THEOREM 2 Along an extremal, all controls $\tau_{1}, \tau_{3}$ and $\tau_{5}$ cannot be singular at the same time.

The next step is to study extremals such that 2 controls are singular at the same time.

THEOREM 3 Along a $\tau_{1}, \tau_{5}$-singular extremal, the control $\tau_{3}$ is bang-bang with at most one switching. Along such extremal, we have that the singular controls are identically $O$ as well as the angular velocity $q$ while the linear velocity $u$ is constant. Moreover, if $\tau_{3}$ has one switching then we can deduce $u \equiv 0$ along the extremal. Furthermore, these extremals are not time optimal.

Proof. Along a $\tau_{1}, \tau_{5}$-singular extremals we must have $\lambda_{u} \equiv 0$ and $\lambda_{q} \equiv 0$. Theorem 2 implies that $\tau_{3}$ cannot be singular. In [2] it is proved that there is at most one switching for the control $\tau_{3}$ and that if there is one we must have $u=q \equiv 0$ and both singular components of the controls are identically zero. Assume now $\tau_{3}$ is constant along the extremal. It follows from (16) and from $\lambda_{u} \equiv 0$ that $q \lambda_{w}=-\lambda_{x} \cos \theta+\lambda_{z} \sin \theta$. Coupled with the fact that $\ddot{\lambda}_{q}=w\left(\lambda_{x} \cos \theta-\lambda_{z} \sin \theta\right)+q w \lambda_{w}-\lambda_{w} \tau_{1} / m \equiv 0$ we obtain $\lambda_{w} \tau_{1}=0$. In particular, since $\lambda_{w} \neq 0$ we have that $\tau_{1}$ is constant and we can differentiate $\lambda_{q}$ up to order 6:

$\lambda_{q}^{(6)}=-4 q^{3}\left(\lambda_{x} \sin \theta+\lambda_{z} \cos \theta\right) \tau_{3}-3 q\left(-2 \lambda_{x} \cos \theta+2 \lambda_{z} \sin \theta-q \lambda_{w}\right) \frac{\tau_{3} \tau_{5}}{m I}$

Using the second derivative of $\lambda_{q}$ and of $\lambda_{u}$, we can reduce the previous expression to $\lambda_{q}^{(6)}=-5 q^{2} \lambda_{w} \frac{\tau_{3} \tau_{5}}{m I}$. However, we must have $\lambda_{q}^{(6)} \equiv 0$. Using the fact that $\tau_{3}$ is constant, we deduce that $q^{2} \tau_{5}=0$. Since moreover $\dot{q}=\tau_{5} / I$, the two last equalities implies that along the extremal we have $\tau_{5} \equiv 0$. Now, if we combine the first and the third derivative of $\lambda_{u}$ we get $2 q^{3} \lambda_{w}=-\left(\lambda_{x} \sin \theta+\lambda_{z} \cos \theta\right) \frac{\tau_{5}}{I}$ which leads to $q \equiv 0$. The equations of motion imply then that $u$ is constant along the extremal and we can deduce that $\ddot{\lambda}_{w} \equiv 0$. For the non optimality of such extremals, one only has to see that they correspond to a pure translational motion that, according to [3], are not optimal.

REMARK 4 This theorem generalizes the results of [2] to the symmetric situation. Moreover, the theorem remains true if we exchange the role of $\tau_{1}$ and $\tau_{3}$ 
Since the non-optimality of the extremals studied in Theorem 3 as been established, let us provide numerically a fastest trajectory. Along a pure translational motion, if we start from $\chi_{0}$ at the origin and follow the $\tau_{1}, \tau_{5}$-singular extremal for 2 seconds, we arrive at $\chi_{f}=(0,0.1,0,0,0.1,0)$. Applying an optimization method to find a time optimal trajectory steering $\chi_{0}$ to $\chi_{f}$ gives a minimum time $t_{f}^{\min } \approx 1.7604 \mathrm{~s}$ which is clearly better than the one of the $\tau_{1}, \tau_{5}$-singular extremal. Figure 2 shows the computed time-optimal trajectory. Figure 2 only shows a singularity of $\tau_{5}$ at the middle of the trajectory which corresponds to the time when the vehicle is properly oriented to use efficiently both its translational thrusters.
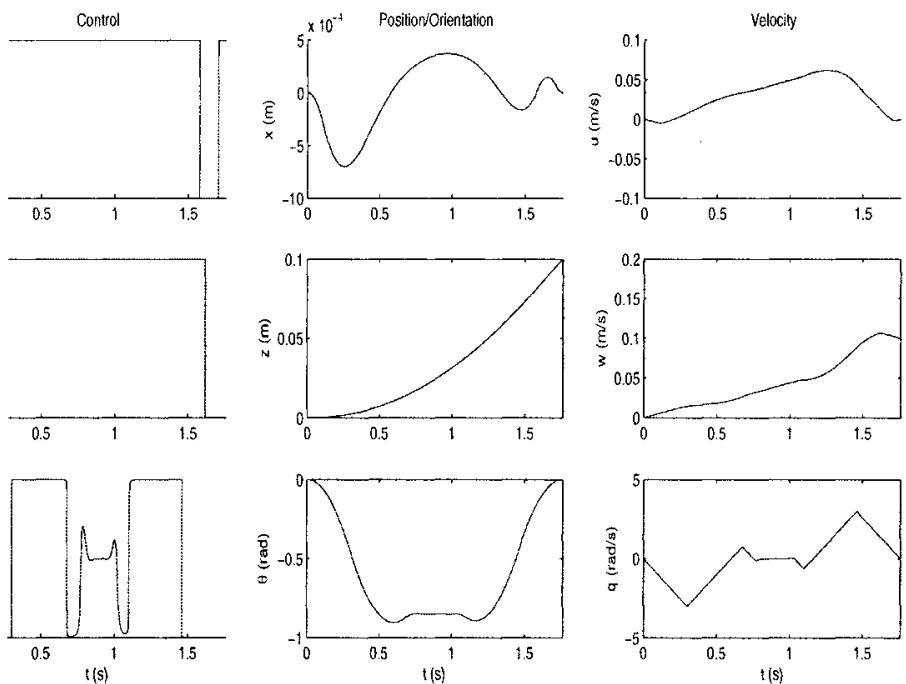

Figure 2. Faster trajectory than a $\tau_{1}, \tau_{5}$-singular extremal

REMARK 5 The optimization method consists in the discretization of the optimal control problem using a Heun integration scheme for the dynamic and discretizing both state and control. Then we write the obtained nonlinear optimization problem in AMPL [6] and apply the large-scale nonlinear optimization software IpOpt [12].

We now turn to the situation when $\tau_{5}$ is bang-bang while the two other controls are singular.

THEOREM 6 Along $a \tau_{1}, \tau_{3}$-singular extremals, the control $\tau_{5}$ is bang-bang with at most one switching.

Proof. This result is a direct consequence of the form of the differential equations for the adjoint vector. 
It could be interesting to exhibit a time optimal trajectory that is $\tau_{1}, \tau_{3}$-singular since it is kind of counter intuitive not to use the full translational power. Actually, if we numerically integrate the Hamiltonian system starting, for instance, from $\chi_{0}=(0,0,0,0,0,0)$, take $\tau_{1}=-\tau_{3}=0.5$ and $\lambda_{x}(0)=\lambda_{z}(0)=$ $\lambda_{u}(0)=\lambda_{w}(0)=0$ we will have a trajectory that is extremal. Additionally, we set $\lambda_{\theta}(0)=0.5$ and $\lambda_{q}(0)=2$ and integrate from $t_{0}=0$ to $T=8 \mathrm{~s}$ to get one switching time for $\tau_{5}$ at $t_{s}=4 \mathrm{~s}$. The switching is from 1 to -1 (because $\left.\lambda_{q}(t)=-\lambda_{\theta}(0) t+\lambda_{q}(0)\right)$. Now, if we use the obtained final configuration $\chi_{T}$ and apply an optimization method to solve the minimum time transfer from $\chi_{0}$ to $\chi_{T}$ we find that the minimum transfer time is $t_{f}^{\min } \approx 8.0005 \mathrm{~s}$ which corresponds to $T$ up to the unavoidable round-off errors. Figure 3 shows the two trajectories.
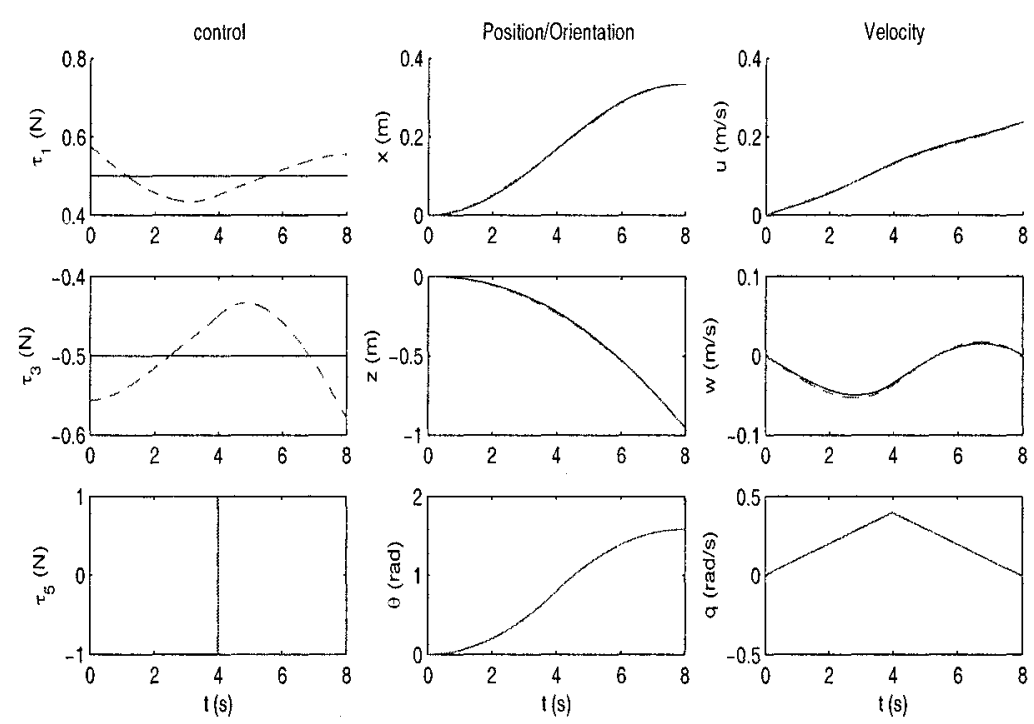

Figure 3. Comparison between the $\tau_{1}, \tau_{3}$-singular trajectory computed by integration of the Hamiltonian system (the dotted one) and the one obtained by application of the nonlinear optimization solver (the plain one).

On Figure 3 one can see that the two trajectories share some similarities but that they are not the same. Indeed, the control strategy is not the same since we do not have $\tau_{1}$ and $\tau_{3}$ constant along the time optimal trajectory computed numerically at the contrary of what was prescribed in our integration of the Hamiltonian system. However, we have a switching at the same time on both trajectories.

Let us now turn to extremals that are singular for one control only. For simplicity we will assume the two other controls to be constant. 
THEOREM 7 Assume $\tau_{3}, \tau_{5}$ to be bang along an extremal. Then, if the extremal is $\tau_{1}$-singular it is of infinite order.

Proof. To determine the order of a $\tau_{1}$-singular extremal, we must find the order of the derivative at which $\tau_{1}$ arises for the first time. A simple computation show that:

$$
\begin{aligned}
& \dot{\lambda}_{u}=-\lambda_{x} \cos \theta+\lambda_{z} \sin \theta-\lambda_{w} q \\
& \ddot{\lambda}_{u}=2 q\left(\lambda_{x} \sin \theta+\lambda_{z} \cos \theta\right)-\lambda_{w} \frac{\tau_{5}}{I}
\end{aligned}
$$

We can easily generalize and see that the only control that can appear in any derivatives of the function $\lambda_{u}$ is the control $\tau_{5}$ and the result follows.

REMARK 8 Theorem 7 still holds if we exchange the role of $\tau_{1}$ and $\tau_{3}$.

Things work differently for the control $\tau_{5}$.

TheOREM 9 Assume $\tau_{1}, \tau_{3}$ to be bang along an extremal. Then, if the extremal is $\tau_{5}$-singular, it is of intrinsic order 2.

Proof. Let us define $H=H_{0}+\varphi_{5} \tau_{5}$. Then, the first two derivatives of the switching function $\varphi_{5}=\lambda_{q} / I$ are given by:

$$
\frac{d \varphi_{5}}{d t}=\left\{H_{0}, \varphi_{5}\right\}, \quad \frac{d^{2} \varphi_{5}}{d t^{2}}=\left\{H_{0},\left\{H_{0}, \varphi_{5}\right\}\right\}+\tau_{5}\left\{\varphi_{5},\left\{H_{0}, \varphi_{5}\right\}\right\} .
$$

Since, $\left\{\varphi_{5},\left\{H_{0}, \varphi_{5}\right\}\right\}=0$, we can keep differentiating and we have

$$
\begin{gathered}
\frac{d^{3} \varphi_{5}}{d t^{3}}=\left\{H_{0},\left\{H_{0},\left\{H_{0}, \varphi_{5}\right\}\right\}\right\} \\
\frac{d^{4} \varphi_{5}}{d t^{4}}=\left\{H_{0},\left\{H_{0},\left\{H_{0},\left\{H_{0}, \varphi_{5}\right\}\right\}\right\}\right\}+\tau_{5}\left\{\varphi_{5},\left\{H_{0},\left\{H_{0},\left\{H_{0}, \varphi_{5}\right\}\right\}\right\}\right\}
\end{gathered}
$$

Explicitely, we have

$$
\ddot{\lambda}_{q}=\frac{-\lambda_{w} \tau_{1}+\lambda_{u} \tau_{3}}{m}, \quad \lambda_{q}^{(4)}=A_{4}+\tau_{5} B_{4}
$$

where $A_{4}=q\left(2 \lambda_{x} \cos \theta-2 \lambda_{z} \sin \theta+q \lambda_{w}\right) \frac{\tau_{1}}{m I}+q\left(2 \lambda_{x} \sin \theta+2 \lambda_{z} \cos \theta-\right.$ $\left.q \lambda_{u}\right) \frac{\tau_{3}}{m I}$ and $B_{4}=-\frac{\lambda_{w} \tau_{3}+\lambda_{u} \tau_{1}}{m I^{2}}$. Since along the extremal the controls $\tau_{1}$ and $\tau_{3}$ are bang, and such that at least one is nonsingular, we have that $B_{4}=-\frac{\left|\lambda_{w}\right|+\left|\lambda_{u}\right|}{m I^{2}}$ is strictly negative. It follows that the extremal is of order 2 and from (25), we have that the order is intrinsic (which means that the coefficient in front of $\tau_{5}$ is identically 0 and not only along the singular extremal). 
REMARK 10 We saw that $B_{4}<0$ so the strict Kelley's necessary condition, [13], for a singular control to be optimal holds.

Following the procedure described in [13] we put the Hamiltonian system (910) under a semi-canonical form in the case of a $\tau_{5}$-singular extremal, the other controls being assumed to be bang. Since this extremal is of intrinsic order two, the four first coordinates of the new system $(\delta, \xi)$ are $\delta=\left(\delta_{1}, \delta_{2}, \delta_{3}, \delta_{4}\right)$ where $\delta_{1}=\lambda_{q} / I, \delta_{2}=\dot{\lambda}_{q} / I=\left(-\lambda_{\theta}+\lambda_{u} w-\lambda_{w} u\right) / I, \delta_{3}=\ddot{\lambda}_{q} / I=$ $\left(-\lambda_{w} \tau_{1}+\lambda_{u} \tau_{3}\right) /(m I), \delta_{4}=\lambda_{q}^{(3)} / I=\left(\left(\lambda_{x} \cos \theta-\lambda_{z} \sin \theta+q \lambda_{w}\right) \tau_{3}-\right.$ $\left.\left(\lambda_{x} \sin \theta+\lambda_{z} \cos \theta-\lambda_{u} q\right) \tau_{1}\right) /(m I)$.

To completely define a new coordinate system we need to find $\xi$ such that the Jacobian $D(\delta, \xi) / D(\chi, \lambda)$ is of full rank. We suggest

$$
\left\{\begin{array}{l}
\xi_{1}=x, \xi_{5}=\lambda_{x} \cos \theta-\lambda_{z} \sin \theta \\
\xi_{2}=z, \xi_{6}=\lambda_{x} \sin \theta+\lambda_{z} \cos \theta \\
\xi_{3}=\theta, \xi_{7}=\lambda_{\theta} \\
\xi_{4}=u, \xi_{8}=\lambda_{u}
\end{array}\right.
$$

The corresponding $D(\delta, \xi) / D(\chi, \lambda)$ is then of full rank and the canonical Hamiltonian system is

$$
\left\{\begin{array}{l}
\dot{\delta}_{1}=\delta_{2}, \quad \dot{\delta}_{2}=\delta_{3}, \quad \dot{\delta}_{3}=\delta_{4} \\
\dot{\delta}_{4}=q\left(2 \xi_{5}+2 \xi_{6}+q \lambda_{w}-q \xi_{8}\right) /(m I)-\left(\xi_{8} \tau_{1}+\lambda_{w} \tau_{3}\right) \tau_{5} /\left(m I^{2}\right) \\
\dot{\xi}_{1}=\xi_{4} \cos \xi_{3}+w \sin \xi_{3}, \quad \dot{\xi}_{2}=w \cos \xi_{3}-\xi_{4} \sin \xi_{8}, \quad \dot{\xi}_{3}=q \\
\dot{\xi}_{4}=-q w+\tau_{1} / m, \quad \dot{\xi}_{5}=-q \xi_{6}, \quad \dot{\xi}_{6}=q \xi_{5} \\
\dot{\xi}_{7}=\xi_{4} \xi_{6}-w \xi_{5}, \quad \dot{\xi}_{8}=-\xi_{5}-\lambda_{w} q
\end{array}\right.
$$

where

$$
\left\{\begin{array}{l}
\lambda_{w}=\left(\xi_{8} \tau_{3}-m I \delta_{3}\right) / \tau_{1} \\
w=\left(\xi_{7}+\lambda_{w} \xi_{4}-I \delta_{2}\right) / \xi_{8} \\
q=\left(m I \delta_{4}-\xi_{5} \tau_{3}+\xi_{6} \tau_{1}\right) /\left(\lambda_{w} \tau_{3}+\xi_{8} \tau_{1}\right)
\end{array}\right.
$$

Since we were able to reduce our system to a semi-canonical form and since from Remark 10 the Kelley's condition holds, it is now possible to apply the results from [13]. In there, the authors describe the behavior of all extremals in the vicinity of the singular manifold $S$ defined by $S=\left\{(\chi, \lambda) \mid \delta_{i}=0, i=\right.$ $1, \cdots, 4\}$. In particular, we can conclude that for each point $\left(\chi_{0}, \lambda_{0}\right)$ in $S$ there exists a 2-dimensional integral manifold of the Hamiltonian system such that the behavior of the solutions inside this manifold is similar to the one of the chattering arcs in the Fuller problem (we have as well the existence of untwisted 
chattering arcs). To be more specific, there is a one-parameter family of solutions of system (27) reaching $\left(\chi_{0}, \lambda_{0}\right)$ in a finite time such that the switching times for the control $\tau_{5}$ are infinite and follow a geometric progression. It is important to notice that this result does not imply the optimality of such trajectories neither it does imply that, assuming $\tau_{1}, \tau_{3}$ constants, every junction between a $\tau_{5}$-singular and a $\tau_{5}$ bang-bang trajectory involves chattering in the control. Indeed, in order for such junction to have chattering the control must be discontinuous, see [5]. this realized for instance of at the junction the angular velocity vanishes: $q=0$. On Figure 4 , we produce an example of a smooth junction between a $\tau_{5}$-singular and a $\tau_{5}$ bang-bang solution. In [4], the reader can see an example of a chattering junction computed in the non symmetrical case.

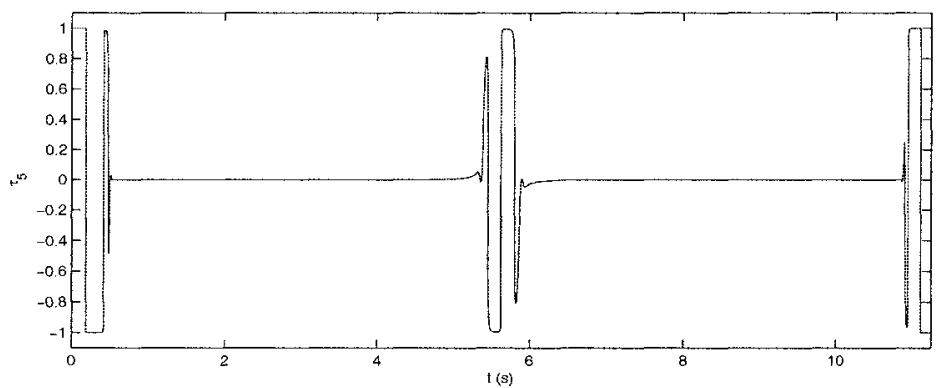

Figure 4. $\tau_{5}$ w.rt. time when singular/bang junctions are continuous.

Figure 4 corresponds to $\chi_{0}$ at the origin and $\chi_{f}=(2,1,0.5,0,0,0)$. Controls $\tau_{1}$ and $\tau_{3}$ are purely bang-bang.

\section{Conclusion}

In this paper, we produce an example of a system involving both, singular extremals of infinite order and singular extremals of intrinsic second order. The existence of chattering extremals has been proved based on a semi-canonical form for our Hamiltonian system along the singular extremals of order 2. In a forthcoming article we will study a more general model of underwater vehicle and will describe precisely the conditions under which a controlled mechanical system has singular extremals of order 2 and then is a potential candidate for chattering solutions. Another direction we intend to take is to use high order necessary condition to discuss the optimality of singular extremals of infinite order. 


\section{References}

[1] M. Chyba, N.E. Leonard, E.D. Sontag. Singular trajectories in the multi-input time-optimal problem: Application to controlled mechanical systems. Journal on Dynamical and Control Systems 9(1):73-88, 2003.

[2] M. Chyba, N.E. Leonard, E.D. Sontag). Optimality for underwater vehicles. In Proceedings of the 40th IEEE Conf. on Decision and Control, Orlando, 2001.

[3] M. Chyba. Underwater vehicles: a surprising non time-optimal path.In 42th IEEE Conf. on Decision and Control, Maui 2003.

[4] M.Chyba, H. Maurer, H.J. Sussmann, G. Vossen. Underwater Vehicles: The Minimum Time Problem. In Proceedings of the 43th IEEE Conf. on Decision and Control, Bahamas, 2004.

[5] J.P. Mcdanell and W.F. Powers. Necessary conditions for joining optimal singular and nonsingular subarcs. SIAM J. Control, 4(2): 161-173, 1971.

[6] R. Fourer, D.M. Gay, B.W. Kernighan. AMPL: A Modeling Language for Mathematical Programming. Duxbury Press, Brooks-Cole Publishing Company, 1993.

[7] T.I.Fossen. Guidance and control of ocean vehicles. Wiley, New York, 1994

[8] A.T. Fuller. Study of an optimum nonlinear control system. J. Electronics Control, 15:6371, 1963.

[9] L.S. Pontryagin, B. Boltyanski, R. Gamkrelidze, E. Michtchenko. The Mathematical Theory of Optimal Processes. Interscience, New-York, 1962.

[10] H.M. Robbins. A generalized Legendre-Clebsh condition for the singular cases of optimal control. IBM J. Res. Develop 11:361-372, 1967.

[11] E.D. Sontag, H.J. Sussmann. Time-Optimal Control of Manipulators. In IEEE Int. Conf: on Robotics and Automation., San Francisco: 1962-1697, 1986.

[12] A. Waechter, L. T. Biegler. On the Implementation of an Interior-Point Filter-Line Search Algorithm for Large-Scale Nonlinear Programming. Research Report RC 23149, IBM T.J. Watson Research Center, Yorktown, New-York.

[13] M.I. Zelikin, V.F. Borisov. Theory of Chattering Control. Birkhäuser, Boston, 1994. 